\author{
Muhamad Abu-Hussein ${ }^{1 *}$, \\ Chlorokostas Georges ${ }^{2}$, Nezar Watted ${ }^{3}$ \\ and Abdulgani Azzaldeen ${ }^{4}$ \\ ${ }^{1}$ University of Naples Federic II, Naples, Italy, \\ Department of Pediatric Dentistry, University of \\ Athens, Greece \\ ${ }^{2}$ Implantologist, Private Dental Practice, Athens, \\ Greece \\ ${ }^{3}$ University Hospital of Würzburg Clinics and \\ Policlinics for Dental, Oral and Maxillofacial \\ Diseases of the Bavarian Julius-Maximilian- \\ University Wuerzburg, Germany and Center for \\ Dentistry research and Aesthetics, Jatt, Israel \\ ${ }^{4}$ Department of Conservative Dentistry, Al-Quds \\ University, Jerusalem, Palestine \\ Dates: Received: 25 October, 2016; Accepted: 31 \\ October, 2016; Published: 01 November, 2016 \\ ${ }^{*}$ Corresponding author: Abu-Hussein Muhamad, \\ DDS, MSc D, MSc, M Dent Sci (Paed Dent), FICD, \\ 123 Argus Street, 10441 Athens, Greece, E-mail: \\ abuhusseinmuhamad@gmail.com \\ www.peertechz.com \\ ISSN: 2455-4634
}

Keywords: Implant stability; ISQ value; Resonance frequency analysis; AL-Technology implant

\section{Research Article \\ A Clinical Study Resonance Frequency Analysis of Stability during the Healing Period}

\section{Introduction}

Osseointegration has been used to define a direct structural and functional connection between ordered living bone and the surface of a load carrying implant [1].

The stability of a dental implant can be defined as the absence of clinical mobility, and this is also the suggested definition of osseointegration. The most important prerequisite for success of ossointegrated dental implants is achievement and maintenance of implant stability. Primary stability is a merely a mechanical phenomenon depending on local bone quality and quantity, surgical preparation technique, and implant design and one of the most important factors in the osseointegration process $[2,3]$.

Initial stabilization is not the same as osseointegration; it must carry the implant during the critical time of the early stages in the development of osseointegration, during which the implant is at risk. The clinical measurement of implant stability and osseointegration is important to be able to assess success in implant dentistry. It is now possible to measure implant stability at any time during the course of implant treatment and loading [2-4].

The primary stability of dental implants depends on the contact of bone with the implant during surgical placement of the implant; this mechanical contact can be obtained with fixation of a press-fit structure into a bony cavity. Primary stability is a major requisite both for direct bone deposit onto the surface of the implant and for subsequent mineral tissue integration $[5,6]$.
If an implant is not stable at the time of installation, micromotion may occur during the healing period, thereby resulting in a thin fibrous layer at the bone-implant interface, an impediment to full osseointegration and a potential harbinger of future implant failure [7] (Figure 1).

Following the placement of an endosseous implant, primary mechanical implant stability is gradually replaced by biologic stability after some weeks post placement. After the initial placement of the implant, there will be a space between the implant surfaces and the bone, and blood clot formation results in the organization of early granulation tissue after four days $[7,8]$. This tissue will give rise to an osteocoating of the implant surfaces, which initiates the processes of biological stability. Thereafter, woven bone in opposition to the implant surface will remodel to form bone within the first 2-4

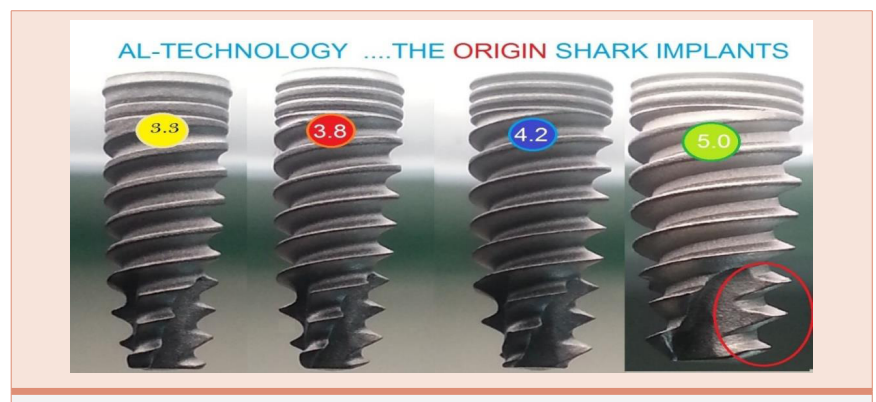

Figure 1: Shark AL-Technology implant system. 
weeks at the osteogenic site of the implant surface. From a clinical standpoint, it is important to realize that during the early weeks of healing, a decrease in implant stability may have to be expected owing to the loss of mechanical stability. Following this period, an increase in clinical stability should be achieved by biological bonding [8-10].

As clinicians have an interest in objectively verifying clinical implant stability to determine the optimal point in time for functional loading, non-invasive methods should be used to assess peri-implant bone height and overall implant stability [7,9].

Resonance frequency analysis (RFA) was initially introduced for use in 1996 for the clinical monitoring of bone formation around an implant as a gauge for its primary stability $[11,12]$. The RFA value can be used not only as an indicator for early diagnosis of the primary stability of an implant, but it can also provide useful messages regarding the secondary stability of an implant. The RFA method is used by the Osstell instrument, which measures the stiffness of the bone/implant interface and is calculated from a resonance frequency generated as a reaction to the oscillation exerted on the implant/ bone system [12-14]. The implant is excited with an oscillating transducer screwed onto the implant, and the resonance specific to the resonance system "implant/bone" is captured electronically over a range of 5-15 KHZ. The implant's own oscillation under a given transducer frequency is dependent upon the character of the implant's bony fixation. This oscillation is of very low amplitude and does not cause any tissue damage during the healing period. The unit of measurement in this approach is the implant stability quotient (ISQ) that is calculated from the resonance frequency. It ranges with increasing stiffness of the interface from 1 to 100 units. A linear relationship exists between the resonance frequency and the ISQ value. An increase of the ISQ by 1 unit appears to correspond to 50 $\mathrm{HZ}$ in resonance. The higher the ISQ, the more stable the implant. To unify the ISQ values, calibration values of each transducer have to be considered, and, for that reason, measurement of different implants cannot be compared to one another, whereas a follow-up of one single implant seems possible and reliable [11-15].

The objective of the present study was to measure the primary stability of Shark AL-Technology implant system during the healing period and determine the factors that affect the Implant Stability Quotient (ISQ).

\section{Materials and Methods}

All patients planned for replacement of missing teeth by Shark AL-Technology implant system were assessed clinically by our clinical team carefully before initiating their treatment [16]. Patients were selected on the basis of the following.

\section{Inclusion criteria}

- $\quad$ Patient age $\geq 19$

- 1 or more missing teeth in either jaw

- Ability and willingness to comply with all study requirements

- Available for clinical follow-up

- Sufficient bone volume with or without localized bone grafting to accommodate implants at least $10 \mathrm{~mm}$ in length
- Absence of clinical or systemic conditions that would contraindicate surgery, implant placement, and/or implant survival

- Adequate oral hygiene

- The insertion torque was equal or greater than $35 \mathrm{Ncm}$ for all the implants.

- All implants used in the study are the Shark AL-Technology implant system having a titanium-alloy threaded-body design with an internal connection feature. Implants were supplied in lengths of $8,10,11.5$, and $13 \mathrm{~mm}$ and diameters of 3.3,3.75,4.2 and $5 \mathrm{~mm}$ (Figure 1).

\section{Exclusion criteria}

- Women who report a current pregnancy

- All patients with chronic diseases like uncontrolled diabetes or psychiatric illness.

- Unrealistic patient expectations of the treatment with respect to esthetic comfort and function.

- Insufficient bone dimension for implants.

- loss or damage of the buccal bone crest ( $>5 \mathrm{~mm}$ ) after extraction of the failing tooth

- need for major bone augmentation procedures with autogenous bone or bone substitutes prior to implant insertion, to obtain an ideal position for the implant (although a minor augmentation procedure to cover exposed threads or interproximal/ buccal grafting owing to hard tissue deficiency was not an exclusion criterion).

- radiotherapy in the maxillofacial region

- treatment with intravenous amino-bisphosphonates

- chemotherapy

- parafunctions (bruxism or clenching).

Patients were seeking treatment to restore the missing teeth created by tooth loss resulting from caries, periodontics, endodontics complications, trauma, and congenital deficiency. The edentulous areas that were to receive the implants, as well as the adjacent structures, were evaluated using an appropriately prescribed combination of periapical, occlusal, panoramic radiographs. Before applying each implant, orthopantomogram was carried out for each patient, sometimes completed with intraoral $\mathrm{x}$-ray images. In order to find out the thickness of alveolus, we made use of dental CT analysis or mapping the gingiva, using a hypodermic needle with a rubber disc [16].

Most patients were instructed on dental hygiene. Recall examinations were carried out according to the previously published scheme.

Dental records for 43 patients who underwent single tooth replacement using Shark AL-Technology implant system at Center for Dentistry, Research \& Aesthetics, Jatt, Almothalat, Israel during a four year period (2010-2012) were retrospectively analyzed. Only 
implants which were functioning for at least three years were included [16].

For the objective of this study, the success rate was recorded according to the criteria suggested by Albredtsson et al. [17], as follows: The unattached implant is immobile when tested clinically, no evidence of periimplant radiolucency appeared radio graphically, vertical bone loss is less than $0.2 \mathrm{~mm}$ annually after the implants' first year of service, absence of persistent and/or irreversible signs and symptoms such as pain, infection, neuropathies, paresthesia, or violation of the mandibular canal [17].

All implants were placed using a conventional, mechanized, nonsubmerged technique, and abundant irrigation with saline solution. Surgical information was collected: age, sex, implant location, implant length and width, bone quality (at the time of surgery) following the anatomic criteria proposed by Lekholm and Zarb (1985), and insertion torque $[16,18]$.

All implants were placed according to the manufacturer recommendations in a non-submerged technique .A healing abutment was placed at the time of surgery to facilitate the reading of the implant stability during the healing period. Surgical information was recorded at the time of the surgery such as age, gender, implant characteristics and bone quality. Implant stability quotient - ISQ was measured using the OssTell Mentor device (OssTell AB Sweden) at $0,2,4,8$ and 12 weeks post-surgery.

Statistical analyses were performed with a mixed effects model using S-Plus 6.0 Professional for Windows. The pertinent mathematical hypotheses were verified in all analyses - statistical significance being considered for $\mathrm{p} \leq 0.05$.

\section{Results}

Out of the total 43 patients, there were 29 females (67\%) and 14 males (33\%). The minimum age of the participants was $19 \mathrm{yrs}$, and the maximum age was 72 yrs 3 months (mean, 44, 3 yrs). 7 patients were smokers. 152 single tooth implants were placed, 114 implants were placed in the posterior region, and 38 were placed in the anterior region. 59 implants were placed in the mandible (39\%) and 93 implants were placed in the maxilla (61\%). Data regarding the number of implants and duration of placement are presented in (Tables 1-3).

All implants were restored with a cemented restoration using the solid conical abutment. The predominant type of implants placed was the solid screw. Regarding the length and diameter of the implants, 28 implants were $3,3 \mathrm{~mm}$ in diameter and $8,10,11.5,13 \mathrm{~mm}$ in length (18\%), 91implants implants were $3,75 \mathrm{~mm}$ in diameter and $8,10,11,5,13 \mathrm{~mm}$ in length(60\%), 18 implants were $4,2 \mathrm{~mm}$ in diameter and $8,10,11.5,13 \mathrm{~mm}$ in length (12\%), and 15 implants were $5 \mathrm{~mm}$ in diameter and 8,10,11.5,13mm in length (10\%).

Among the total 152 implants, the location implant maxillary were 6 (7\%)implants central incisor, 14(15\%) implants lateral incisors, 4 (4\%)implants canine, 31(33\%) implants premolar, and 38 (41\%)implants molar. The location implants mandibular were 3(5\%) implants central incisor, 5(8\%) implants lateral incisors,6 (10\%) implants canine, 24(41\%) implants premolar, and 21(36\%) implants molar (Figure 2).
Table 1: Implant distribution by length and diameters and the area of placement

\begin{tabular}{|l|l|l|l|l|l|l|l|l|}
\hline Diameter & \multicolumn{3}{|c|}{ Length/mm } & $\begin{array}{l}\text { No. of } \\
\text { implants }\end{array}$ & \multicolumn{2}{|c|}{$\begin{array}{c}\text { Area of } \\
\text { placement }\end{array}$} \\
\hline & $8 \mathrm{~mm}$ & $10 \mathrm{~mm}$ & $11.5 \mathrm{~mm}$ & $13 \mathrm{~mm}$ & & & anterior & posterior \\
\hline 3,3 & 2 & 4 & 11 & 11 & 28 & $18 \%$ & 18 & 10 \\
\hline 3,75 & 4 & 11 & 40 & 36 & 91 & $60 \%$ & 16 & 75 \\
\hline 4,2 & 4 & 4 & 5 & 5 & 18 & $12 \%$ & 4 & 14 \\
\hline 5,0 & 6 & 1 & 6 & 2 & 15 & $10 \%$ & 0 & 15 \\
\hline & & & & & 152 & $100 \%$ & 38 & 114 \\
\hline
\end{tabular}

Table2: Number of implants max and mand $(\mathrm{N}=152)$.

\begin{tabular}{|l|l|l|}
\hline $\mathbf{N}=152$ & amount & $\%$ \\
\hline Max. & 93 & $61 \%$ \\
\hline Mand. & 59 & $39 \%$ \\
\hline Total & 152 & $100 \%$ \\
\hline
\end{tabular}

Table 3: Number of implants maxillary and mandibular.

\begin{tabular}{|l|l|l|l|}
\hline Zone & Max. & Mand. & Total \\
\hline anterior & 24 & 14 & 38 \\
\hline posterior & 69 & 45 & 114 \\
\hline Total & 93 & 59 & 152 \\
\hline
\end{tabular}

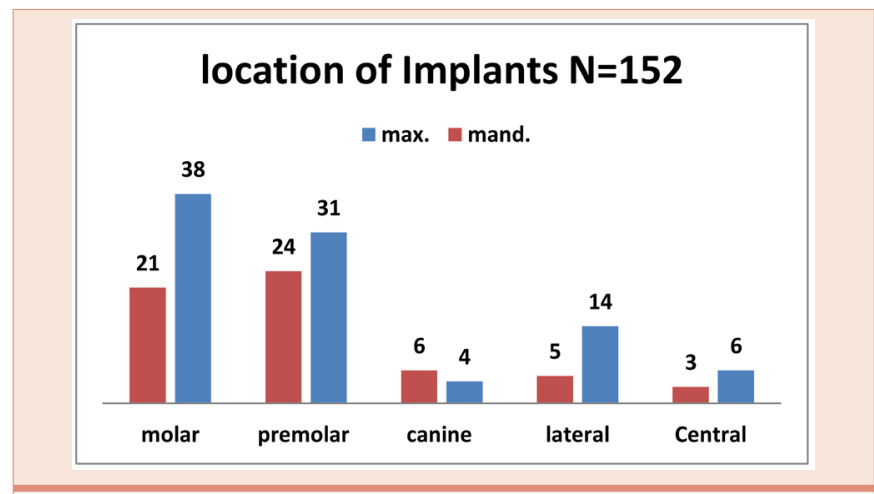

Figure 2: The location of the Implants maxillary and mandibular.

From 152 implants, 24 implants were placed in anterior maxilla, 69 implants in the posterior maxilla, 14 implants in the anterior mandible, and 45 implants in the posterior mandibular, Based on the sampling frame and the sample dimension, 152 values of the ISQ measurements were recorded in Table for each case.

The arithmetic mean was used to describe the centre of the data, for this study. The raw data was summarized and reorganized using the following formula:

$$
\text { Sample Mean } \bar{x}=\frac{1}{n} \sum_{i=1}^{n} a_{i}
$$

Where: $\mathrm{N}=152$ represents the number of implants; $\mathrm{Xi}=\mathrm{ISQ}$ values.

In these diagrams the ISQ mean values are the dependent variables, which depend to some degree on the other variable, also 
called the independent variable, in this case "Number of weeks after surgery".

The mean ISQ of all the implants was 72, 18 on the day of the surgery. The lowest ISQ value of the implants was at 4 weeks after the surgery in both bone types with a mean value of 63,2 . After that the implant stability increased up to 12 weeks post-surgery, reaching a value of 73.02. (Table 4),(Chart 1).

The ISQ value of the implants placed in female patients were higher than the ISQ values of the ones placed in male patients (Chart 2).

With respect to the location of the implants the IQS was lower in the maxillary arch than in the mandibular arch, it was also higher in the anterior areas of the arch than in the posterior areas, which corresponds with different bone types found in the different locations of the maxillary and mandibular arch (Chart 3).

No statistically significant relationship was found between the diameter, length and the insertion torque and the resonance frequency analysis.

\section{Discussion}

Implant stability is a key factor for the success of implant therapy). At placement, adequate primary stability of an implant is essential to allow undisturbed bone healing while thereafter, secondary stability, result of the osseointegration process, permits an optimal distribution of the functional loads through the bone implant interface $[19,20]$. Given the importance of this parameter the attention to its measure in the clinical practice has always been high. The first methods used to clinically evaluate implant stability were the tapping method, radiography and the Periotest. However, all these methods lack enough precision and repeatability in quantifying stability, therefore a precise and repeatable measure of implant stability was needed [20,21].

In 1994 Meredith introduced in the field of implant dentistry a vibration nondestructive testing method: resonance frequency $(\mathrm{RF})$ [22].

Since 1999 this method of analysis has been commercially available as the Osstell equipment (Integration Diagnostics, Göteborg, Sweden). The technique is based on a small L-shaped transducer that contains two piezoelectric elements and is screwed on top of the implant or its abutment [21]. The first element is excited in the range of $5-15 \mathrm{KHz}$ thus transmitting a microscopic and harmless vibration to the bone implant interface, the response to this vibration is then registered by the second element, transmitted through an output cable to a frequency/response analyzer and transformed into an Implant Stability Quotient (ISQ). Receiving the output of the equipment in the form of ISQ is a great advantage over the experimental equipment that was used until 1999 and gave back a result that was expressed in $\mathrm{Hz}$ and in addition needed calibration if measures taken with different transducers were to be compared. Conversely, ISQ units are automatically calibrated for each different transducer and therefore easily and directly comparable. The ISQ units range from 1 to 100 with higher values indicating better stabilities. Today the Osstell has been updated with a new version called Mentor (Integration

\begin{tabular}{|l|l|l|l|l|l|}
\hline \multicolumn{3}{|c|}{ Table 4: The calculated mean values of ISQ measurements. } \\
\hline \multicolumn{2}{|c|}{} & & \multicolumn{3}{c|}{ ISQ MEAN } \\
\hline Weeks after surgery & Total & Men & Gonder & \multicolumn{2}{|c|}{ Dental arch } \\
\hline 0 & 72,18 & 71,26 & 73,8 & 70,66 & 73,6 \\
\hline 2 & 66,12 & 60,78 & 72,36 & 72,28 & 60,1 \\
\hline 4 & 63,2 & 62,5 & 67,38 & 66,34 & 61,86 \\
\hline 8 & 69,6 & 68,74 & 73,1 & 74,18 & 67,86 \\
\hline 12 & 73,02 & 71,87 & 75,6 & 75,8 & 70,1 \\
\hline
\end{tabular}

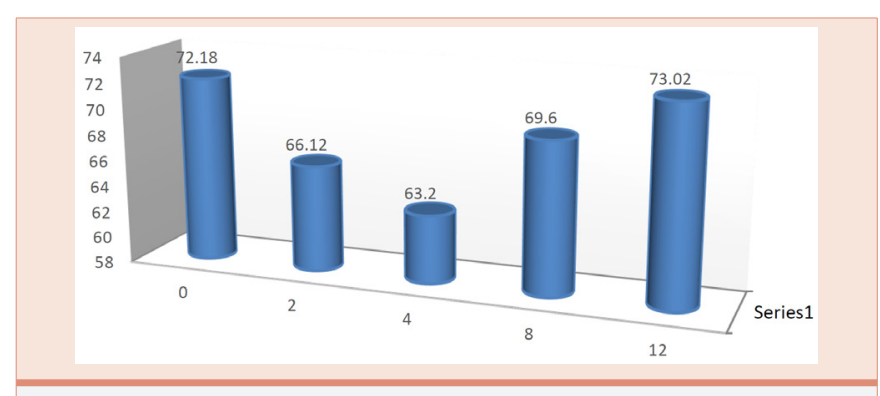

Chart 1: Relationship between ISQ and time during study.

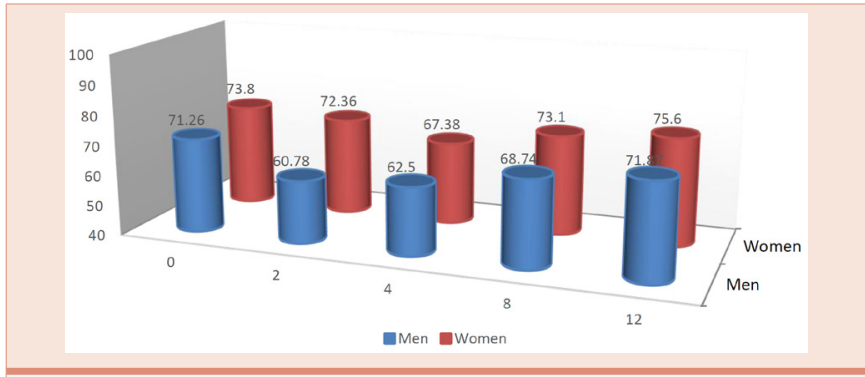

Chart 2: Relationship between ISQ and gender.

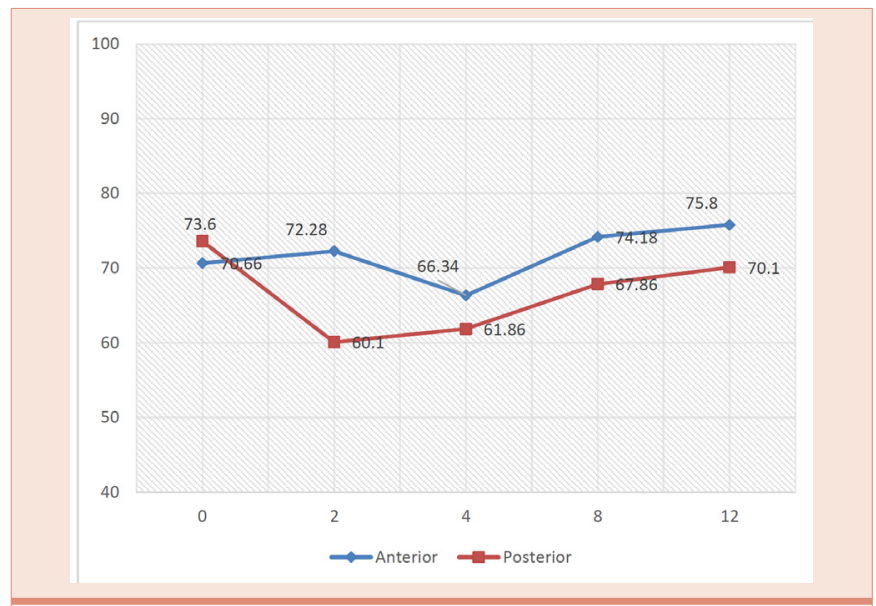

Chart 3: Relationship between ISQ and dental arch position.

Diagnostics) that, while maintaining the ISQ scale, features wireless transducers that are electromagnetically excited [19-23].

Dental implants are a successful treatment modality for missing teeth. There are several ways to evaluate the bone-implant interface. Invasive methods, like the amount of torque required to remove an 
implant, have been used in animal studies [16,24-27]. Clearly, this is a destructive method in which the application of shear stress at the implant interface leads to failure; therefore, it is not applicable for clinical assessment [28-32]. Cutting torque measurement is a clinical method that uses cutting resistance measurements during threading of implants and has been used by several investigators to identify bone densities during implant placement [16,24,27,30,32]. Although this technique provides an assessment of bone quality at the time of placement, it does not allow for any direct measurement of the changes that influence the supporting bone over time. RFA is a noninvasive objective testing modality of implant stability, which is a useful tool to establish implant loading time [30-32]. The quantity and location of cortical and trabecular bone surrounding the implant are important factors in stability as they contribute to bone-implant contact $[28,30,31,32]$ Nedir et al., observed that the majority of implants in the maxilla and mandible had ISQ values of $<60$ and $>60$, respectively. Consistent with the results of our study, Barewal et al., and Bischof et al., found higher values in the mandible, with statistically significant differences in the latter $[3,4]$.

During the process of the osseointegration of the implants the value of the ISQ varies with time. At the surgical phase the average ISQ for all the implants was 72,18 indicating a high primary stability, higher than the results obtained by different clinicians using different type of implants Bishoff et al. [34], found an average of 60.3, Boronat Lopez et al. 62.6 [7]. In our study the value of the ISQ decreased to 63,2 at 4 weeks post insertion. However all the clinicians cited above found a decrease in the ISQ during week 3 and four of healing. After that there was a steady increase in the ISQ value up to 12 weeks post insertion when it reached a mean ISQ value of 73,02 . It may be speculated that the decrease of the ISQ values may be due to the loss of mechanical stability identified during the early phase of healing and bone remodeling $[7,34]$.

With respect to the gender of the patients our clinical study showed a higher ISQ value for the female patients compared to the male patients. These results were also confirmed by different other clinicians [7-11].

Gender comparison revealed higher RFA-measured stability in male versus female patients [8], whereas other authors described significantly higher values in female patients [7]. A possible explanation for this variation in the reported results was suggested by Seong et al., who attributed these deviations to the age differences among the study groups [35]. Study groups characterized by older mean ages may possibly include more postmenopausal women, and therefore lower ISQ values should be expected due to worse bone density [36]. This difference in bone density between women and men was also established by a long- term study conducted by Östman et al. [5]. However, they concluded that these findings were not clinically relevant as there were no differences in the failure rates between men and women reported so far [35].

Huber et al., who reported that whereas several studies produce an apparent sex difference in the first place, this difference disappear when taking into account the effects of other variables, which is also in line with the results of the present study. Supporting further this multivariate statistical model, Huber et al., identified significantly lower ISQ values for implants in the edentulous maxilla of female patients at various time points while this gender difference disappeared when RFA measurements were compared in the edentulous mandible. It therefore seems that surgical related factors such as jaw influenced more the RFA measurements of these studies than sex [10].

In the light of the above, it seems safe to conclude that patientrelated variables (i.e. sex and age) are not significantly effective in implant stability according to ISQ values [11].

Initial implant stability measured by Osstell device varied significantly among anatomical regions of the maxilla and the mandible. Data reported so far in the foreign literature demonstrate that mandibular implants have significantly higher initial stability than maxillary implants, which is also in line with the results presented in this study $[5,16]$.

The correlation between ISQ values and the bone type of the regions where implants were inserted was further investigated by Friberg et al. who assessed bone density by cutting torque measurements when placing implants and showed that decreasing implant stability was seen with decreasing bone quality. This finding has been attributed by several authors to the presence/absence of cortical bone, which is 10 to 20 times stiffer than trabecular bone, which most likely also explains differences between mandibular and maxillary implants, since maxillary bone is often softer owing to lesser extents of cortical bone [8-11] (Chart 3).

Bischof et al., Further investigated the parameters regarding the implant stability quotient after 12 weeks of healing, right before the abutment connection to proceed with the prosthetic restoration. The mean ISQ at this time point in the mandible was still higher than in the maxilla but the difference between bone type, which was a determinant for the initial ISQ measurements, was leveled out. The authors proposed that the latter may be explained by bone densification of the soft bone surrounding the implants. In our study, however, the ISQ discrepancy of the two jaws remained at the same levels for both time points ( $\mathrm{p}>0.05)$ [34].

The lower success rates reported in the posterior area when compared with the anterior region can also be explained in terms of bone density, and, as expected, ISQ values were recorded lower for the posterior area [34].

Östman et al. (2006), found that implant stability was higher in posterior than in anterior regions, in spite of the fact that implant placement generally is regarded as more challenging in posterior regions because of the anticipated more frequent presence of soft bone quality. However, these contradictory results can be partially explained by the fact that all wide implants in their study, which showed higher ISQ values than RP/NP implants, were placed in posterior regions [5].

Seong et al. (2008), indicated that there were no statistical significant ISQ differences between anterior and posterior regions. However, we believe that each region is characterized by different bone quality and, therefore, conclusions drawn from comparisons between anterior and posterior regions are of questionable validity. 
Hence, current Osstell measurements were used to determine the mean ISQ values of all four regions, and statistical analysis of our data revealed that posterior maxillary implants are the least stable, which is also in line with the results reported by Seong [35].

All the clinicians cited above found a decrease in the ISQ during week 3 and four of healing. After that there was a steady increase in the ISQ value up to 12 weeks post insertion when it reached a mean ISQ value of 73,02 It may be speculated that the decreaseof the ISQ values may be due to the loss of mechanical stability identified during the early phase of healing and bone remodeling $[7,8]$.

Regarding the location of the implant, the ISQ values were greater in the mandible than in the maxilla. The mean value of the ISQ for the mandible being $>60$, while in the maxilla $<60$. Furthermore the implants placed the anterior part of the mouth either in the maxilla or in the mandible showed higher values than those placed in the posterior areas of the mouth. In the anterior part of the mouth the bone has a thick cortical plate and a dense trabecular bone compared to the posterior areas of the mouth and therefore the primary stability is higher in these regions of the mouth [19-22].

The bone quality and implant stability are lower in posterior areas; for this reason the posterior implant success rate is less than the anterior. In the anterior area, the thick cortical and the dense trabecular bone will increase primary stability; in this study, ISQ was higher in this area than the posterior region. Some authors suggest that using longer and wider implants increases primary stability due to the increased bone-implant contact surface area $[18,21-23,25]$.

Our study has also shown the difference of ISQ values between implants placed in the maxilla and those placed in the mandible. Although the ISQ was lower in the maxilla, the difference was not significant (Figure 3).

Other authors have also shown with Brånemark implants that implant stability was higher in the mandible than in the maxilla while implant length did not affect implant stability [10-13].

This data corresponds with our results Regarding location of the implant, the ISQ was greater in the mandible than in the maxilla, with mean values of 71, 26 on the day of surgery and after 12 weeks was 71,87 , respectively. There were no significant differences between the anterior and posterior sectors. On relating the location and position of the implants, the ISQ values were found to be higher in the posterior upper jaw than in the anterior sector of the maxilla, while in the case

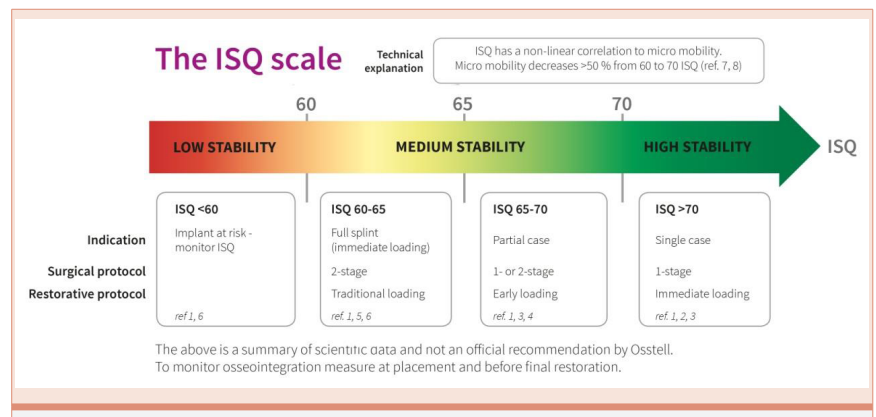

Figure 3: The ISQ Scale Model Osstell. of the mandible no significant differences were recorded between the anterior and posterior sectors.

Regarding implant position (anterior or posterior), we found no differences - in coincidence with the data published by Balleri et al., who studied the same parameter after one year of loading. In our study, on relating the location and the position of the implants, the ISQ values were found to be greater in the upper posterior sector than in the upper anterior zone, while no differences were noted in the case of the lower jaw [36].

The stability quotient measured immediately after implant placement was greater for the larger diameter and shorter length designs, in mandibular implants, and in implants inserted in more compact bone.

\section{Conclusion}

Resonant frequency analysis is probably the most objective and reliable method of measuring implant micromobility in various stages of implant therapy. Implant stability is clinically proven to play an important role in the efficiency of treatment, providing the insight into implant therapy outcome.

Implant therapy success depends on biomechanical stability factors data. Good implant stability reduces the risk of failure. Recent researches have shown that high values of resonant frequency analysis indicate therapy success with a minimal probability of failure.

These findings may have important implications related to immediate or early loading protocols. Further research is needed to expand upon the results of this study.

Research employing a greater number of macrothread designs placed in a variety of clinical scenarios would be beneficial towards the goals of optimizing implant macrothread design and determining if RFA technology holds predictive value in immediate loading success rates.

\section{References}

1. Branemark PI (1985) Introduction to osseointegration. In: Bra ${ }^{\circ}$ nemark PI, Zarb GA, Albrektsson T (1985) Tissue-Integrated Prostheses: Osseointegration in Clinical Dentistry. Chicago III: Quintessence 611-612.

2. Albrektsson T, Wennerberg A (2004) Oral implant surfaces: Part 1--review focusing on topographic and chemical properties of different surfaces and in vivo responses to them. Int J Prosthodont 17: 536-543.

3. Friberg B, Sennerby L, Roos J, Lekholm U (1995) Identification of bone quality in conjunction with insertion of titanium implants. A pilot study in jaw autopsy specimens. Clin Oral Implants Res 6: 213-219.

4. Friberg, B, Sennerby L, Linden B, Grondahl K, Lekholm U (1999a) Stability measurements of one-stage Branemark implants during healing in mandibles. A clinical resonance frequency analysis study. Int J Oral Maxillofac Surg 28: 266-272.

5. Ostman PO, Hellman M, Wendelhag I, Sennerby L (2006) Resonance frequency analysis measurements of implants at placement surgery. Int $\mathrm{J}$ Prosthodont 19: 77-78.

6. Brochu JF, Anderson JD, Zarb GA (2005) The influence of early loading on bony crest height and stability, a pilot study. Int J prosthodont 18: 506-512.

7. Lopez B, Martinez JB, Pelayo JL (2008) Resonance frequency analysis of dental implant stability during healing period. Med Oral Circ Bucal 13: E244-E247. 
8. Balshi SF, Allen FD, Wolfinger GJ, Balshi TJ (2005) A resonance frequency analysis assessment of maxillary and mandibular immediately loaded implants. Int J Oral Maxillofac Implants 20: 584-594.

9. Quesada-García MP, Prados-Sánchez E, Olmedo-Gaya MV, Muñoz-Soto E, Vallecillo-Capilla M, Bravo M (2012) Dental implant stability is influenced by implant diameter and localization and by the use of plasma rich in growth factors. J Oral Maxillofac Surg 70: 2761-2767.

10. Huber S, Rentsch-Kollàr A, Grogg F, Katsoulis J, Mericske R (2012) A 1 -year controlled clinical trial of immediate implants placed in fresh extraction sockets: stability measurements and crestal bone level changes. Clin Implant Dent Relat Res. 14: 491-500.

11. Rokn A, Ghahroudi AR, Mesgarzadeh A, Miremadi A, Yaghoobi S (2011) Evaluation of stability changes in tapered and parallel wall implants: a human clinical trial. J Dent (Tehran) 8: 186-200.

12. Bajoghli F, Sabouhi M, Davoudi A, Badrian H, Molazem M (2015) A brief review on contemporary methods and equipment used for implant stability assessments. J Int Oral Health 7: 10-12.

13. Zix J, Hug S, Kessler-Liechti G, Mericske-Stern R (2008) Measurement of dental implant stability by resonance frequency analysis and damping capacity assessment: Comparison of both techniques in a clinical trial. Int $\mathrm{J}$ Oral Maxillofac Implants 23: 525-530.

14. Al-Jetaily S, Al-Dosari AA (2011) Assessment of osstell ${ }^{\mathrm{TM}}$ and periotest systems in measuring dental implant stability (in vitro study). Saudi Dent $\mathrm{J}$ 2011 23: 17-21.

15. Gupta RK, Padmanabhan TV (2011) Resonance frequency analysis. Indian J Dent Res 22: 567-573.

16. Abu-Hussein M, Watted N, Shamir D (2016) A Retrospective Study of the AL Technology Implant System used for Single-Tooth Replacement. Int J Oral Craniofac Sci 2: 039-046.

17. Albrektsson $T$ (1998) A multicenter report on osseointegrated oral implants. $J$ Prosthet Dent 60: 75-84

18. Lekholm U, Zarb GA (1985) Patient selection and preparation. In: Branemark PI, Zarb GA, Albrektsson T (eds). Tissue-Integrated Prostheses: Osseointegration in Clinical Dentistry. Chicago: Quintessence 199-209.

19. Meredith N (1998) Assessment of implant stability as a prognostic determinant. Int J Prosthodont 11: 491-501.

20. Meredith N (1997) On the clinical measurement of implant stability and osseointegration. Thesis, Goteborg University.

21. Meredith N, Cawley P, Alleyne D (1996) Quantitative determination of the stability of the implant-tissue interface using resonance frequency analysis. Clin Oral Impl Res 7: 261-267.

22. Meredith N, Cawley P, Alleyne D (1994) The application of modal vibration analysis to study bone healing in vivo. J Dent Res 73: 793-798.

23. Meredith N, Shagaldi F, Alleyne D, Sennerby L, Cawley P (1997) The application of resonance frequency measurements to study the stability of titanium implants during healing in the rabbit tibia. Clin Oral Impl Res 8: 234 243.

24. Abu-Hussein M, Azzaldeen A, Aspasia SA, Nikos K (2013) Implants into fresh extraction site: A literature review, case immediate placement report. J Dent Implant 3: 160-164.

25. Abu-Hussein M., Abdulgani A., Watted N .Zahalka M (2015) Congenitally Missing Lateral Incisor with Orthodontics, Bone Grafting and Single-Tooth Implant: A Case Report. Journal of Dental and Medical Sciences 14: 124-130.

26. Abdulgani A, Kontoes N, Chlorokostas G, Abu-Hussein (2015) Interdisciplinary Management Of Maxillary Lateral Incisors Agenesis With Mini Implant Prostheses: A Case Report. Journal of Dental and Medical Sciences 14: 3642.

27. Abusalih A, Ismail H, Abdulgani A, Chlorokostas G, Abu- Hussein M (2016) Interdisciplinary Management of Congenitally Agenesis Maxillary Lateral Incisors: Orthodontic/Prosthodontic Perspectives, Journal of Dental and Medical Sciences 15: 90-99

28. Abdulgani A, Kontoes N, Chlorokostas G, Abu-Hussein M (2015) Interdisciplinary Management Of Maxillary Lateral Incisors Agenesis With Mini Implant Prostheses: A Case Report. IOSR-JDMS 14: 36-42.

29. Abu-Hussein M, Chlorokostas G, Watted N, Abdulgani A, Jabareen A (2016) Pre-Prosthetic Orthodontic Implant for Management of Congenitally Unerupted Lateral Incisors - A Case Report. Journal of Dental and Medical Sciences 15: 99-104.

30. Muhamad AH, Azzaldeen A, Nezar W, Mohammed Z (2015) Esthetic Evaluation of Implants Placed after Orthodontic Treatment in Patients with Congenitally Missing Lateral Incisors. J Adv Med Dent Scie Res 3: 110-118.

31. Abdulgani M, Abdulgani AZ, Abu-Hussein M (2016) Two Treatment Approaches for Missing Maxillary Lateral Incisors: A Case. Journal of Dental and Medical Sciences 7: 78-85.

32. Muhamad Abu-Hussein et al. (2016) Congenitally Missing Lateral Incisors; Orthodontic, Restorative, and Implant Approaches. Int J Dent 2: 2: 71-84.

33. Barewal RM, Oates TW, Meredith N, Cochran DL (2003) Resonance frequency measurement of implant stability in vivo on implants with a sandblasted and acid-etched surface. Int J Oral Maxillo-fac Implants 18: 641 651.

34. Bischof M, Nedir R, Szmukler-Moncler S, Bernard JP, Samson J (2004) Implant stability measurement of delayed and immediately loaded implants during healing. Clin Oral Implants Res 15: 529-539.

35. Seong WJ, Holte JE, Holtan JR, Olin PS, Hodges JS, et al. (2008) Initial stability measurement of dental implants placed in different anatomical regions of fresh human cadaver jawbone. J Prosthet Dent 99 425-434.

36. Balleri P, Cozzolino A, Ghelli L, Momicchioli G, Varriale A (2002) Stability measurements of osseointegrated implants using Osstell ${ }^{\mathrm{TM}}$ in partially edentulous jaws after 1 year of loading: a pilot study. Clin Implant Dent Relat Res 4: 128-132.

Copyright: (C) 2016 Abu-Hussein M, et al. This is an open-access article distributed under the terms of the Creative Commons Attribution License, which permits unrestricted use, distribution, and reproduction in any medium, provided the original author and source are credited. 